\title{
Distribución espacial de Exogyra boussingaulti de la formación Tibú- Mercedes (Cretácico: Aptiano-Albiano) en el Municipio de San Andrés (Santander: Colombia)
}

Spatial distribution of Exogyra boussingaulti of the TibúMercedes Formation (Cretaceous: Aptian-Albian) in the Municipality of San Andrés (Santander: Colombia)
Autor:

Javier H. Jerez-Jaimes ${ }^{1}$

Eliana X. Narváez-Parra ${ }^{2}$

Juan Camilo Martínez Peñuela ${ }^{3}$

Fabián Zarza Rincón ${ }^{3}$

Weymar Alonso Bautista Caballero ${ }^{3}$

Anamaría Quintero Valencia ${ }^{3}$

\section{INVESTIGACIÓN CIENTÍFICA}

Cómo citar este artículo:

Jerez-Jaimes JH., Narváez-Parra, EX., MartínezPeñuela JC., Zarza-Rincón F., Bautista-Caballero W.Ay Quintero-Valencia A. Distribución espacial de Exogyra boussingaulti de la Formación TibúMercedes (Cretácico: Aptiano-Albiano) en el Municipio de San Andrés (Santander, Colombia), Innovaciencia 2020; 8(1): 1-8

DOI: https://doi.org/10.15649/2346075X.817

Fecha de recepción:

Recibido: 01 May 2020

Aceptado: 30 August 2019

Publicado: 01 December 2020

Palabras clave:

Distribución agrupada, Índice varianzamedia, Índice de Green, Índice de dispersión de Morisita.

Keywords:

Clump distribution, variance to mean ratio, Green's coefficient, Morisita index of dispersion.

\section{RESUMEN}

Exogyra boussingaulti fue un bivalvo reclinante de sedimentos blandos, encontrado en la formación Tibú-Mercedes del Cretácico inferior en el Municipio de San Andrés (Santander: Colombia). Sobre la ecología de E. boussingaulti se conoce muy poco en aspectos de reproducción, dispersión o depredación, por esta razón, se determinó la distribución espacial de esta especie mediante el cálculo de tres índices de dispersión a partir de los datos obtenidos de una cuadricula elaborada en un bloque rodado de $2 \mathrm{~m}^{2}$. Con base en los resultados, fue posible inferir que E. boussingaulti presentaba una distribución en parches no concentrados determinados por su ciclo reproductivo y limitados por la competencia por alimento a nivel intraespecífico. De igual forma este artículo se presenta como una estrategia de enseñanza que contribuye con el proceso educativo de los estudiantes de ciencias geológicas y con la solución a preguntas pequeñas en paleobiología.

\section{ABSTRACT}

Exogyra boussingaulti was a reclining bivalve of soft sediments found in the Tibú-Mercedes Formation of the Lower Cretaceous in the Municipality of San Andrés (Santander: Colombia). On the ecology of E. boussingaulti very little is known in reproduction, dispersal or predation aspects, for this reason, it was determined the spatial distribution of this species through the calculation of three indices of dispersion from the data obtained from a grid drawn in a block of $2 \mathrm{~m}^{2}$. With this results was possible to infer that E. boussingaulti had a distribution in unconcentrated patches (Clumps) determined probably by its reproductive cycle and limited by competition for food at the intraspecific level. In the same way, this article is presented as a teaching strategy that contributes to the educational process of geological sciences students and with the solution to short questions in paleobiology.

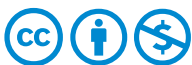

CC BY-NC 4.0
M.Sc. Biología. Paleobiólogo Independiente. Autor de correspondencia: javjerez@gmail.com

2 M.Sc. Biología. Profesora. Facultad de Ciencias Exactas, Naturales y Agropecuarias, Universidad de Santander UDES. enarvaez@udes.edu.co

3 Estudiantes del Programa de Geología, Universidad de Santander UDES. 


\section{INTRODUCCIÓN}

Esta publicación es de carácter académico y científico, pues pretende servir de guía para el trabajo en el campo y contribuye con información sobre los patrones de distribución del bivalvo fósil Exogyra boussingaulti.

La paleoecología trata de reconstruir las interacciones de los organismos con su medio ambiente y sus componentes biológicos y fisicoquímicos. Dentro de nuestro ejercicio como maestros en este campo disciplinar partimos de la necesidad de conocer sobre estos aspectos propios de cada especie, línea de la ecología que se conoce como autoecología. Como organismo modelo para aprender a reconstruir aspectos autoecológicos utilizamos a Exogyra boussingaulti, un bivalvo que fue descrito por Alcide d'Orbigny en 1842 a partir de ejemplares recolectados por Juan Bautista Boussingault en la margen derecha del rio Sube en el municipio del Socorro y también en el municipio de Matanza del Departamento de Santander (Colombia) como producto de las exploraciones iniciales para la creación de la Escuela de Minas durante el periodo de 1823 a $1833^{(1)}$.

Taxonomía de Exogyra boussingaulti

Clase Bivalvia

Orden Ostreida

Familia Griphaeidae

Género Exogyra

Especie Exogyra boussingaulti d'ORBIGNY, 1842

La descripción original de Orbigny ${ }^{(2)}$ para Exogyra boussingaulti fue:

"E. testá elongato-oblongá, irregulari, valvá inferiori subcarinatá, late plicata; unco laterali contorto; valvá superiori carinatá, elevatá, plicatá." (latín)
La descripción de especies es un ejercicio que se ha venido perdiendo con el tiempo y es considerado por nosotros como un error, pues ¿cómo se puede identificar si no se sabe describir? Si bien es cierto, las especies actuales pueden ser identificadas por su ADN como barcodes o códigos de barras, las especies que ya no existen y que no dejaron registro de su ADN dependen de nuestra habilidad descriptiva para diferenciarlas y llevarlas a la luz del conocimiento. Debido a la ausencia de descripciones y precisiones en el reporte de las especies al describir depósitos o formaciones geológicas se genera confusión al momento de establecer un dialogo de saberes sobre la paleoecología de las especies. Para el área de estudio en el municipio de San Andrés (Santander) Acosta ${ }^{(3)}$ en su estratigrafía de García Rovira registra a Exogyra boussingaulti en la quebrada Isgaura del municipio de San Andrés en 1960, posteriormente Caballero

y Sierra ${ }^{(4)}$ en 1991 en su tesis sobre la Estratigrafía del Cretácico en el área de San Andrés Santander determinan a Exogyra squamata como un elemento característico de la Formación Tibú-Mercedes (Intervalo 1) que aflora en los márgenes de la Quebrada Isgaura. No es de extrañar que las especies de Exogyra pudiesen coexistir espacial y temporalmente, pero en nuestro trabajo de campo nos basamos en las descripciones geológicas más actualizadas y se puede caer en el error en el que nuestro grupo se encontró, dar por hecho que la especie de Exogyra estudiada correspondía con la registrada. En este caso Caballero y Sierra ${ }^{(4)}$ observaron a Exogyra boussingaulti confundiéndola con Exogyra squamata. A pesar de la antigüedad de las descripciones de las especies y los registros nominales en las descripciones estratigráficas se consideró necesario explicar de manera visual la diferencia entre las dos especies. La Figura 1 se elaboró con los dibujos originales del Alcide d'Orbigne ${ }^{(2)}$ en su Coquilles et Equinodermes Fossiles de Colombie en 1842, publicación basada en las colectas de especímenes de Boussingault durante 1821 a 1833. 

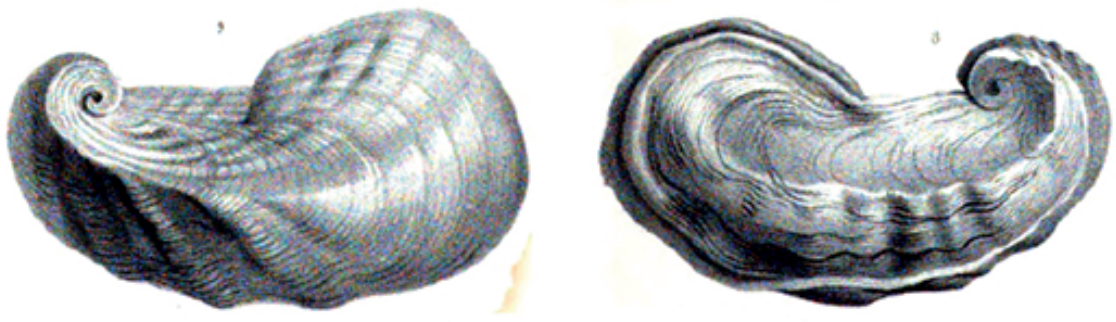

\section{Exogyra boussingaulti}

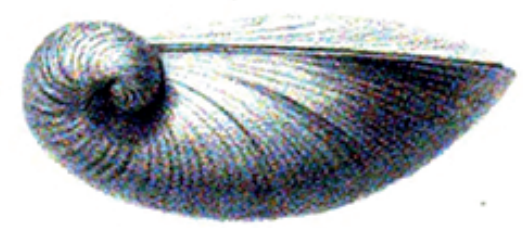

Exogyra squamata

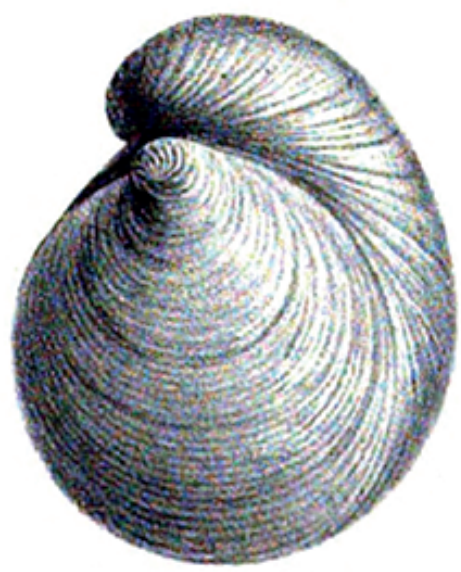

Figura 1. Morfología externa de las conchas de Exogyra boussingaulti y E. squamata. Nótese la presencia de costillas gruesas en E. boussingaulti y de líneas finas sobre la concha de E. squamata. Las figuras de la izquierda muestran las especies en posición en vida. Tomadas de Alcide d'Orbigne ${ }^{(2)}$.

Ecología de Exogyra boussingaulti

Fijación al sustrato: Bivalvo Reclinado

Los Grifaeidae vivían en la superficie de sustratos blandos, esencialmente suspendidos en los sedimentos ${ }^{(5)(6)}$. A este hábito Stanley ${ }^{(7)}$ denominó reclinable. El descenso de las poblaciones a través del Paleozoico se atribuye a la evolución de grupos de organismos que perturbaron y desestabilizaron los sedimentos ${ }^{(8)}$.

Exogyra boussingaulti fue un bivalvo que yacía sobre el sedimento sin la producción de bisos o cementos. Para facilitar este modo de vida, la concha de esta especie era más gruesa e inequivalva, es decir, una valva es más grande y de diferente forma a la otra que era más pequeña, para estabilizarse y resistir la corriente desarrolló costillas gruesas. La Alimentación se da por filtración del agua y debido a su modo sésilpasivo se denomina por suspensión, en las que aprovechan las zonas con niveles de energía del agua altos, es decir, zonas donde las corrientes permiten el arrastre de los nutrientes hasta el lugar de fijación de los organismos.

La ecología de los bivalvos fósiles es muy importante para comprender patrones de sedimentación, energía del ambiente o batimetría. Como parte del proceso de aprendizaje y análisis de datos paleo ecológicos, nos propusimos aprovechar la oportunidad de conocer el patrón espacial de dispersión de Exogyra boussingaulti en un bloque rodado en el lecho del rio Listara, proveniente de la formación Tibú-Mercedes (Cretácico: Aptiano-Albiano) en el municipio de San Andrés (Santander: Colombia).

\section{MATERIALES Y MÉTODOS}

\section{Área de estudio:}

Durante la salida de campo de Paleontología de la Universidad de Santander UDES al municipio de San Andrés (Santander) en la provincia de García Rovira, durante el segundo semestre de 2019, se realizó una exploración en los depósitos del lecho 
del rio Listara $\left(\mathrm{N}^{\circ}, 782353^{\circ}\right.$ y W- $\left.72^{\circ}, 842428\right)$ en la que se observaron cantos y bloques de gran tamaño $>2 \mathrm{~m}^{2}$ de diferentes tipos de materiales: arenosos, calizas y lutitas (shale), en su mayoría con evidencia fosilífera (Conchas) (Figura 2, A, B y C).

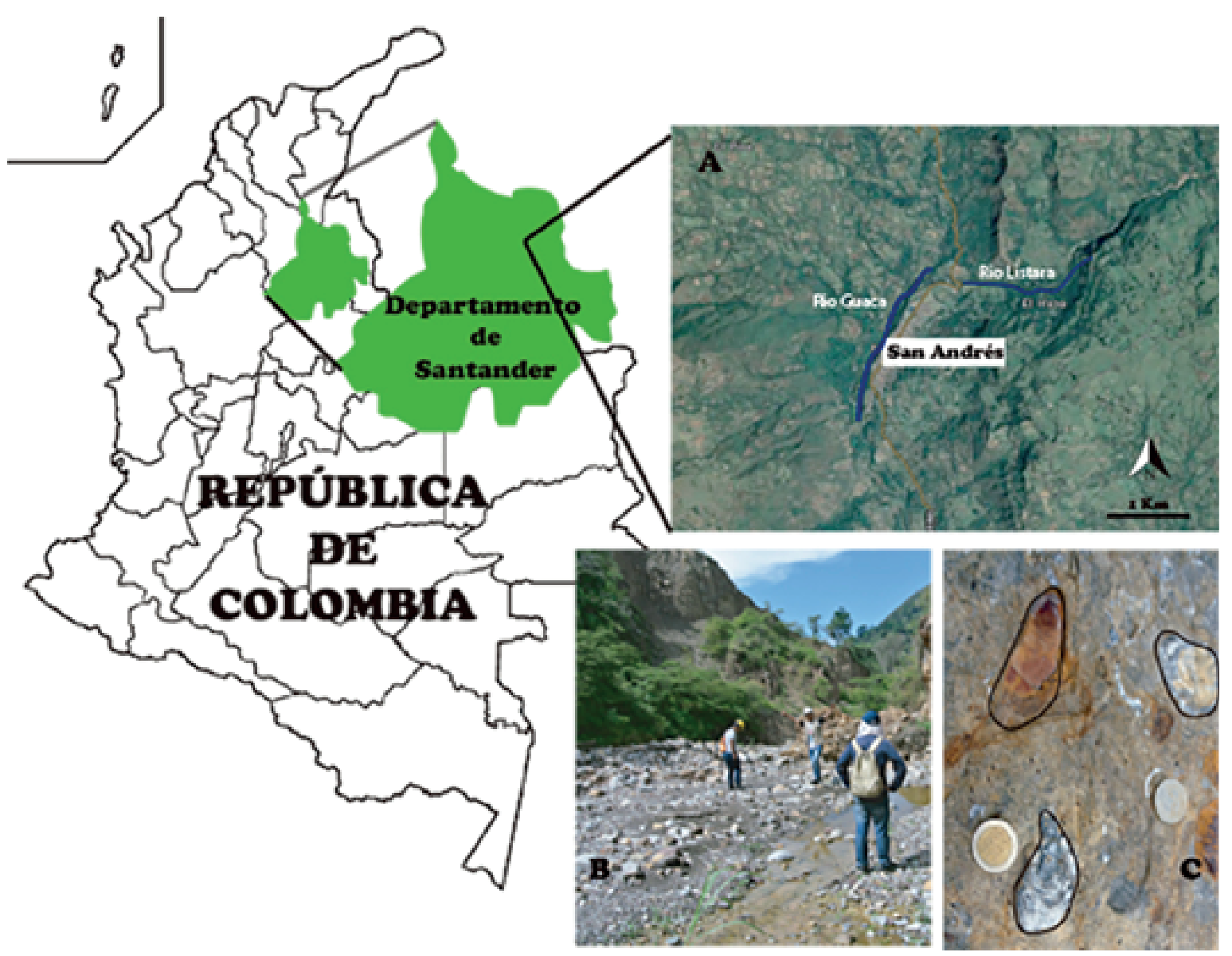

Figura 2. Ubicación del área de estudio. A, Municipio de San Andrés. B, Lecho del rio Listara. C, Exogyras en posición de vida.

\section{Formación de origen}

La formación Tibú- Mercedes Kitm; está ubicada en la parte basal del rio Guaca y márgenes del rio Listara. Esta formación en el municipio de San Andrés se caracteriza por el contenido de rocas sedimentarias, para una mayor descripción de la formación se recomienda revisar "Estratigrafía del Cretácico en el área de San Andrés Santander" de Caballero y Sierra (4).

\section{Bloque para análisis}

Durante el recorrido por el cauce del rio Listara se encontraron varios bloques de calizas de gran tamaño que contenían comunidades del bivalvo Exogyra boussingaulti la mayoría en posición en vida. Se eligió un bloque horizontal de $2 \mathrm{~m}^{2}$ sobre el cual se trazó con cinta de papel un cuadrante que fue dividido en seis subcuadrantes de igual tamaño. Se contó el número de Exogyras en cada cuadrante y se registraron los datos en las libretas de campo (Figura 3). 

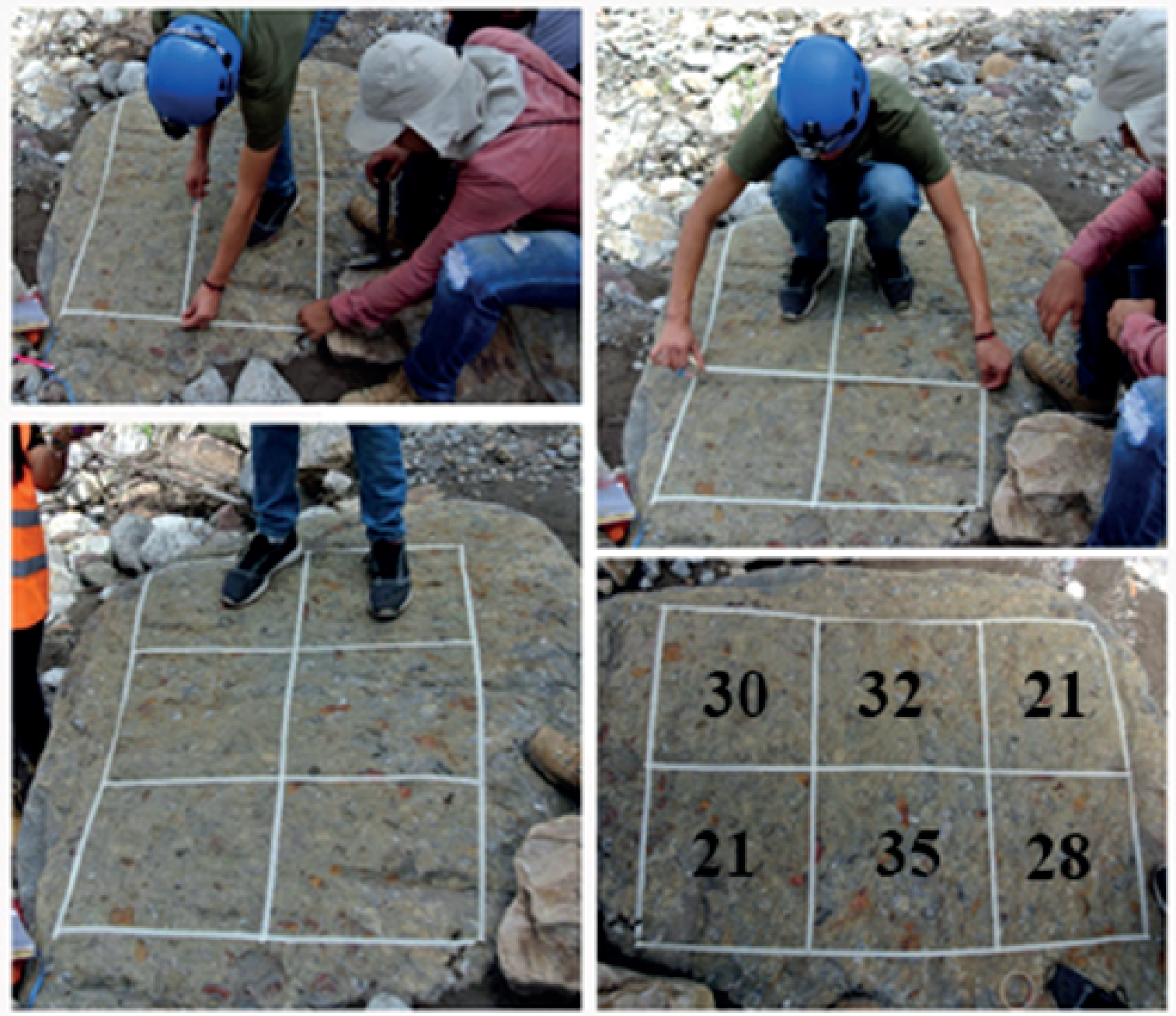

Figura 3. Elaboración de la cuadricula para conteo ecológico de E. boussingaulti. Nótese el número de individuos en cada cuadrante.

Análisis de distribución de Exogyra boussingaulti

Para determinar el modelo de distribución ecológica de Exogyra boussingaulti se calcularon los índices de dispersión de la Relación Varianza sobre la Media, el coeficiente de Green y el índice de dispersión de Morisita y sus respectivos límites (Tabla 1) según la metodología de Krebs ${ }^{(9)}$.

Tabla 1. Valores límite calculados a partir de $\operatorname{Krebs}^{\left({ }^{(9)}\right.}$

\begin{tabular}{lccc}
\hline Índice de dispersión & Uniformidad & Azar & Agregación \\
\hline Varianza/media & 0 & 1 & $>1$ \\
Coeficiente de Green & $-0,006$ & 0 & 1 \\
Coeficiente de Morisita & 0,97 & 0 & $\mathrm{n}$ \\
\hline
\end{tabular}

\section{RESULTADOS}

A partir de la cuadricula (grid) elaborada en campo (Figura 3) se obtuvieron los conteos de E. boussingaulti en cada cuadrante, el número de individuos por cuadrante se aprecia en la Figura 3, un total de 167 individuos se contabilizaron en posición natural. La tabla 2 muestra los valores calculados y su agrupación estimada. 
Tabla 2. Resultados obtenidos del cálculo de los tres índices de dispersión.

\begin{tabular}{lcc}
\hline Índice de dispersión & Azar & Agregación \\
\hline Varianza/media & --- & 1,2 \\
Coeficiente de Green & 0,001 & --- \\
Coeficiente de Morisita & 0,06 (estandarizado) & 1 \\
\hline
\end{tabular}

\section{DISCUSIÓN}

Los valores obtenidos de los índices de dispersión (Tabla 2) indican que E. boussingaulti formaba comunidades que compartían espacios pequeños, pero los índices muestran una débil tendencia al modelo agrupado en parches (clumps) y una tendencia a una distribución aleatoria. En este punto debe hacerse la diferenciación entre los modelos agrupados en los bivalvos reclinados tipo Exogyra (Aetostreon) couloni de la formación Rosablanca del Cretácico inferior en el municipio de Zapatoca (Santander: Colombia) con una cobertura total del sustrato, por capas de individuos, utilizando el máximo del espacio disponible, siendo un modelo conglomerático o de camas, producto del mecanismo de reproducción (Obs. Pers. Javier Jerez-Jaimes), similar a la actual Crassostrea virginica que puede formar arrecifes de decenas a miles de metros en longitud ${ }^{(10)}$. Muy diferente con el modelo encontrado en Exogyra boussingaulti, el cual forma grupos pequeños (Clumps) con individuos separados sin una cobertura total del sustrato.

Una explicación a este modelo observado puede ajustarse a la hipótesis propuesta por Powel et al. (11) para comunidades actuales de ostras en Texas donde "la mortalidad producida por la depredación y la enfermedad modulada por la competencia por alimento, permite que las ostras grandes afecten la supervivencia de las ostras que viven en parches adyacentes". La figura 4, explica la hipótesis propuesta en este estudio de la formación de los parches o clumps por competencia de alimento entre Exogyras de mayor tamaño con las de menor tamaño que terminan por morir sin poderse fijar al sustrato, formando de esta manera espacios entre los parches, observados en el registro paleontológico. Los valores obtenidos con los índices de dispersión por esta razón apuntan a una distribución azarosa no contagiosa dentro del clump (Figura 2C), eso explica la discrepancia entre los índices calculados. También es una evidencia clara del problema del tamaño del cuadrante, por lo que es recomendable ensayar con diferentes tamaños del mismo. La experiencia en invertebrados del profesor Jerez-Jaimes en estudios ecológicos indica que se deben realizar los conteos con tres tamaños proporcionales al tipo y tamaño de invertebrado. Siendo este un ejercicio pedagógico en paleontología vale la pena que se considere esta recomendación para estudios posteriores. De igual forma se debe considerar que en esta experiencia no se relacionaron los tamaños de las Exogyra boussingaulti información que hubiese permitido una mayor comprensión de la dispersión de la especie y otros aspectos ecológicos. Esto no quiere decir que este halla sido un estudio mal diseñado desde el punto de vista de la enseñanza de las ciencias, ya que el origen de la pregunta surge de la exploración preliminar de estos depósitos en el municipio de San Andrés, es decir, la pregunta surge en el campo y se utilizan los recursos disponibles (en especial tiempo) para tratar de recolectar el máximo de información del hallazgo. Con la información disponible se pudo determinar y llegar a conocer la dispersión de una especie extinta en su hábitat, se plantean hipótesis que regulan ese mecanismo, se aprende sobre la biología de una especie y se aprende a resolver preguntas científicas. 


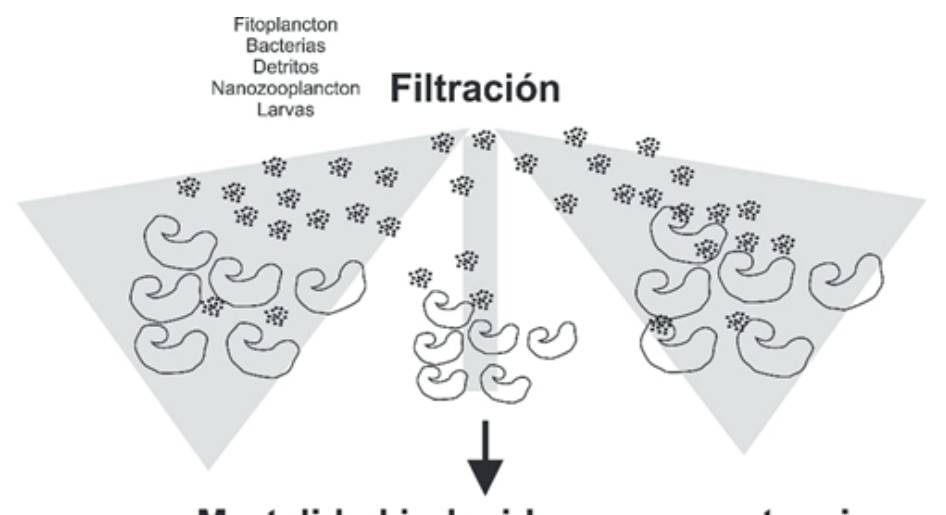

Mortalidad inducida por competencia

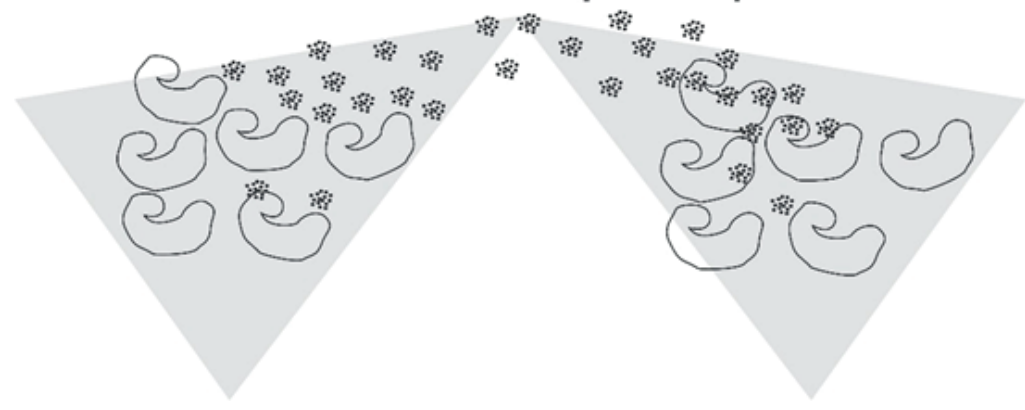

Figura 4. Modelo de formación de parches (Clumps) en Exogyra boussingaulti en la formación Tibú-Mercedes (Cretácico inferior).

Se establece que en algunos momentos existía una continuidad en la cobertura del sustrato por Exogyras grandes (pioneras) y pequeñas (colonizadoras). La capacidad de los individuos grandes para captar mayor concentración del material particulado suspendido, limita la cantidad de alimento para las jóvenes colonizadoras, que al permanecer en niveles nutricionales bajos podrían haber sido más sensibles a las enfermedades y a la depredación, siendo sustraídas del sustrato y dando origen a parches no colonizados entre los grupos de mayor tamaño. Javier H. Jerez-Jaimes.

La importancia del modelo de dispersión es crucial para determinar los rangos de distribución de las especies, la estructura genética (de especies actuales) y la historia evolutiva. Especies que se reproducen sin un estado larval pueden únicamente dispersarse por flotación resultando en modelos de distribución espacial en parches a diferencia de las especies con estados larvales de dispersión ${ }^{(12)}$. Esta afirmación no es absoluta ya que el estudio del cual surge la afirmación se refiere a especies de los diferentes grupos de moluscos. El modo de dispersión larval planctónica en bivalvos también pude dar origen a la formación de poblaciones en parches. Según Malchus ${ }^{(13)}$ en su estudio filogenético sobre larvas de bivalvos, establece que los Griphaeinae taxón al que pertenece Exogyra boussingaulti presentaron larvas velígeras planctónicas- planctotróficas, que al parecer no cubrían grandes distancias, pero que sí las alejaban de la población parental, explicando de nuevo el por qué de la débil diferenciación entre una dispersión al azar y una agrupada para esta especie. La figura 5 permite entender el posible ciclo reproductivo de Exogyra boussingaulti, considerando una fertilización externa y un desarrollo embrionario y larval en la columna de agua hasta su descenso y colonización del sustrato, este modelo se basó en los estados de desarrollo de la ostra actual Ostrea edulis descritos por Acarli y Lok ${ }^{(14)}$. 


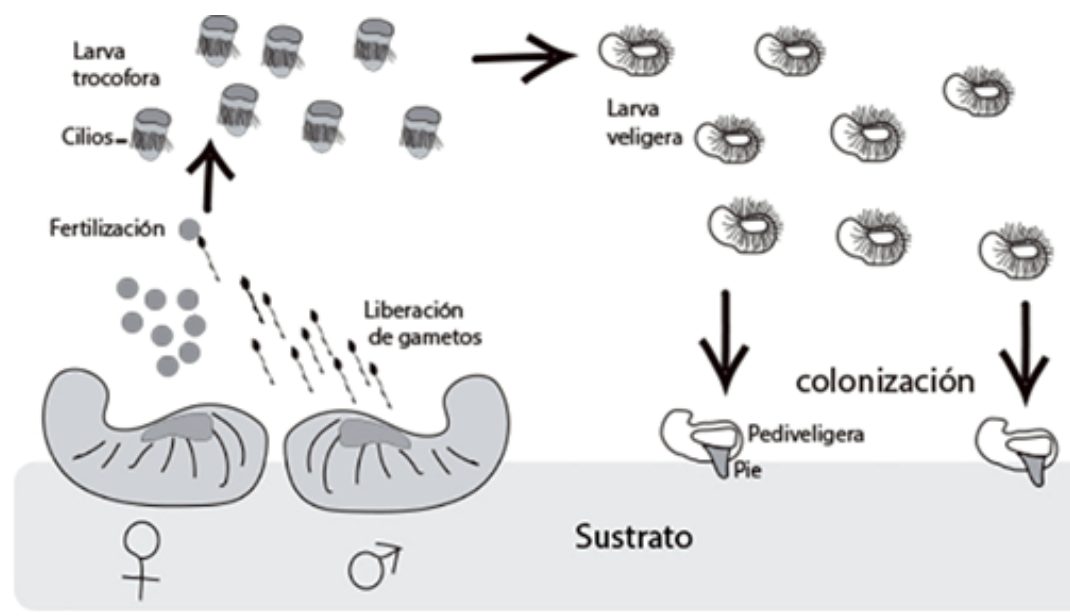

Figura 5. Ciclo reproductivo hipotético de Exogyra boussingaulti. La larva velígera planctónica facilita la dispersión de los juveniles alejándolos de los parentales, a una distancia relativamente corta, limitada por el desarrollo a las fases de colonización de sustrato. Javier H. Jerez-Jaimes.

\section{CONCLUSIONES}

Se cumplió con el objetivo de enseñanza del proceso de investigación científica, con la debida aplicación del método científico, desde la observación en campo y la formulación de preguntas actuales no resueltas que contribuyen al conocimiento paleontológico y paleobiológico de la región, así como el desarrollo de métodos para obtener y analizar la información, finalizando con la divulgación de este conocimiento. Se justificó la importancia de leer las descripciones de las especies para confirmar su determinación publicada en documentos geológicos más recientes y disponibles, en contraposición a lo planteado por uno de los pares evaluadores de este artículo quien planteaba la inutilidad de este proceso.

Los índices utilizados para determinar la distribución espacial de Exogyra boussingaulti permitieron establecer que se ajusta a un modelo de agrupaciones en parches no contagiosos (juntos o continuos) con individuos al azar.

La distribución espacial de E. boussingaulti permitió construir la hipótesis del establecimiento de los parches poblacionales no contagiosos ni continuos.

Se propone que dicha distribución en parches con individuos al azar fue consecuencia de la competencia por alimento y de su ciclo de desarrollo reproductivo.

\section{AGRADECIMIENTOS}

Los autores expresan sus agradecimientos a los pares evaluadores por sus comentarios para la publicación de esta experiencia pedagógica y científica.

\section{REFERENCIAS}

1. Espinosa, B. A. Historia de las investigaciones geológicas en Colombia. Notas a partir de la segunda mitad del siglo XIX. En: Arboleda L C, Arias de Greiff J y Espinosa Baquero A. Historia Social de la ciencia en Colombia. Tomo II. Matemáticas, Astronomía y Geología. Colciencias. 1993.

2. D'Orbigny A. Coquilles et Echinodermes Fossiles de Colombie, recueillis par M. Boussingault, 65 ps., 6 pl., París;1842.

3. Acosta C J M. Estratigrafía de García Rovira. Boletín de Geología. No 5. Universidad Industrial de Santander. 1960.

4. Caballero OV, Sierra RH. Estratigrafía del Cretácico en el área de San Andrés Santander. Tesis de Pregrado. Universidad Industrial de Santander, Escuela de Geología; 1991.

5. Hallam A. Morphology, palaeoecology, and evolution of the genus Gryphaea in the British Lias. Philosophical Transactions of the Royal Society London B. 1968. 254:91-128. https://doi.org/10.1098/rstb.1968.0014 
6. Stenzel HB. Oysters. In: Moore, R. C. and C. Teichert, eds. Treatise on Invertebrate Paleontology, Part N, vol. 3 (Bivalvia). 271 pp. Geological Society of America, Inc. Lawrence, Kansas; 1971.

7. Stanley SM. Relation of Shell Form to Life Habits of the Bivalvia (Mollusca). Geological Society of America, Inc. Memoir 125; 1970. https://doi.org/10.1130/MEM125-p1

8. Abdelhand A, Elewa AMT. Evolution of the Upper Cretaceous Oysters: Traditional morphometrics approach. Chapter 6. In: Elewa AMT. (ed.), Morphometrics for Nonmorphometricians. Lecture Notes in Earth Sciences 124. Springer-Verlag; 2010. https:/ / doi.org/10.1007/978-3-540-95853-6_6

9. Krebs CJ. Ecological Methodology. Second edition. Adisson-Wesley; 1999.

10. Dame RF. 1976. Energy Flow in an intertidal oyster population. Estuarine Coastal Marine Science.1976.4: 243-253.

https://doi.org/10.1016/0302-3524(76)90058-X
11. Powell EN, Cummins H, Stantos RJ, Staff G. Estimation of the size of Molluscan larval settlement using the death assemblage. Estuarine Coastal Shelf Science. 1984. 18: 367-384. https:/ / doi.org/10.1016/0272-7714(84)90078-7

12. Johnson MP, Allcock AL, Pye SE, Chambers SJ, Fitton DM. The effects of dispersal mode on the spatial distribution patterns of intertidal molluscs. Journal of Animal Ecology. 2001.70: 641-649.

https:// doi.org/10.1046/j.1365-2656.2001.00519.x

13. Malchus N. Early ontogeny of Jurassic bakevelliids and their bearing on bivalve evolution. Acta Paleontológica Polónica. 2004. 49 (1): 85-110.

14. Acarli S y Lok A. Larvae Development Stages of the European Flat Oyster (Ostrea edulis). The Israeli Journal of Aquaculture - Bamidgeh 61(2);114-120. 2009. 